\title{
Improved diagnostic policy for respiratory tract infections essential for patient management in the emergency department
}

Randy Poelman ${ }^{1}$, Johan van der Meer², Corina van der Spek ${ }^{3}$, Annelies Riezebos-Brilman ${ }^{1,4}$, Marjolein Knoester ${ }^{1}$, Coretta Van Leer-Buter ${ }^{1}$, Alexander W Friedrich ${ }^{1}$ \& Hubert G Niesters*,1

${ }^{1}$ The University of Groningen, University Medical Center Groningen, Department of Medical Microbiology \& Infection Prevention, Division of Clinical Virology, Groningen, The Netherlands

${ }^{2}$ University Medical Center Groningen, Emergency Department, Groningen, The Netherlands

${ }^{3}$ University Medical Center Groningen, University Medical Center Staff, Groningen, The Netherlands

${ }^{4}$ Currently: University Medical Center Utrecht, Department of Medical Microbiology, Utrecht, The Netherlands

*Author for correspondence: Tel.: +31 50361 5060; h.g.m.niesters@umcg.nl

\begin{abstract}
Aim: Establishing an optimal diagnostic policy for patients with respiratory tract infections, at the emergency department (ED) of a university hospital in The Netherlands. Methods: Adult patients were sampled at admission, during the respiratory season (2014-2015). The FilmArray-RP was implemented at the clinical virology laboratory. Diagnostics were provided from 8 am to 10 pm, weekends included. Results: 436/492 $(89 \%)$ results were available while patients were still at the ED. Median TAT from admission to test result was 165 min (IQR: 138-214). No antibiotics were prescribed in 94/207 (45\%) patients who tested positive for a virus. 185/330 (56\%) hospitalized patients did not need admission with isolation measures. The value-based measure, expressed in euro-hour (€h), increased to tenfold compared with previous policy. Conclusion: An optimal policy is essential for patient management, by providing timely, reliable diagnostics.
\end{abstract}

First draft submitted: 26 April 2019; Accepted for publication: 24 March 2020; Published online: 3 June 2020

Keywords: clinical virology • co-creation • diagnostic stewardship • emergency department • patient management - point-of-care testing $\bullet$ respiratory tract infections

Molecular diagnostics for infectious diseases is important in a clinical setting for disease management and infection control. During the last decades, PCR-based technologies increased the capability to detect pathogens and results were available within the same or next day when maintaining a proper diagnostic policy [1]. From a clinical perspective, timely detection is essential for patient management, definitely at the emergency department (ED) with a narrow window of opportunity.

No treatment options are available for most respiratory viruses. As a result, rapid diagnostics for respiratory viral infections are often focused on testing for influenza virus only, and additionally for respiratory syncytial virus (RSV) mostly in children. Although antigen testing is still frequently used, molecular testing is currently the gold standard. Not only for influenza virus and RSV, but for a larger syndromic panel of viral and bacterial respiratory targets, causing lower and upper respiratory tract infections (LRTI and URTI) [2].

According to the integrated stewardship model [3], diagnostic stewardship involves the performance of proper diagnostic testing that has immediate effect on hospitalization, patient isolation and antimicrobial therapy. This requires clinical laboratories to maintain and enhance their diagnostic assays in order to detect a variety of clinically important respiratory pathogens and adapt it to the regional and local microbial epidemiology. This has become evident during the enterovirus D68 upsurges in Europe [4,5]. Additionally, the criteria for diagnostic assays must meet high performance standards [6]. Good sensitivity and specificity of the assay, as well as the ability to detect pathogens within a short period of time (preferably while the patient is still in the ED), are necessary to have an impact on clinical management like therapeutic interventions, disease management and infection prevention [7-9].

Future Medicine 


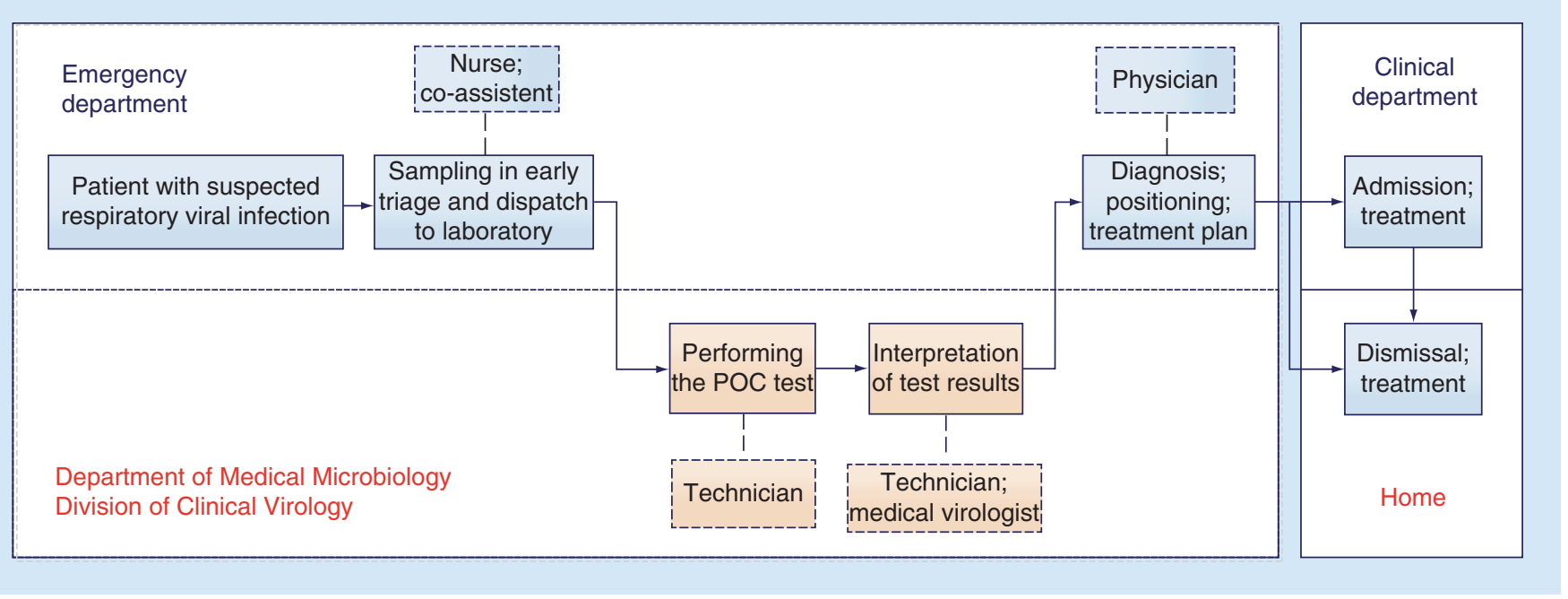

Figure 1. The schematic presentation of our optimal diagnostic policy for respiratory viral infections in patients that were admitted to the emergency department.

POC: Point-of-care.

Tertiary care hospitals have the challenge that a vast amount of isolation rooms are reserved for specific patient groups like transplantation or oncology patients. However, especially during respiratory seasons, an increased number of patients present themselves at the ED. These patients are admitted to an isolation room in order to prevent potential spreading of a viral infection, until the diagnostic test results are available. This delay has a negative impact on the patient flow through the hospital, as well as a negative financial impact because beds are blocked for other patients and healthcare productivity decreases [10]. Bed management (occupancy) could be improved with appropriate and rapid diagnostics while the patient is still present at the ED, to prevent unnecessary isolation and to enable adequate cohorting of patients.

Currently, rapid, sensitive and specific amplification-based assays have become available shifting the trend from single-target testing to multiplex syndromic respiratory testing, as well as from centralized to decentralized pointof-care (POC) testing [1,11-13]. The use of these assays for respiratory targets has been described before and the clinical benefit is mainly due to the decreased turnaround times (TAT) and the large number of targets that can be detected. However, questions on comprehensiveness, reliability and financial impact (cost-benefit) remains [14]. Therefore, the aim of this study was to create an optimal diagnostic policy for respiratory viral infections including the implementation of a rapid syndromic diagnostic assay. With a critical window of opportunity in a hospitalwide setting, we also assessed the value of rapid diagnostic testing for patients with respiratory viral infections at the ED. We therefore have conceptualized the so-called euro-hour (€h) model, to describe and discuss the financial impact [3].

\section{Methods}

The diagnostic routing starts with patient admission at the ED and includes timely sampling and dispatching the samples to the clinical virology laboratory close to the ED in the same hospital, using a rapid tubing system. The diagnostic assay is performed in the clinical virology laboratory, which assures an optimal controlled setting. Reporting the diagnostic result to the physician at the ED completes the diagnostic routing.

The improved diagnostic policy (see Figure 1) was characterized by three main measures throughout the diagnostic chain in order to increase diagnostic impact by reducing the TAT. First, sampling of patients with respiratory symptoms in early stage of triage, meeting the criteria (see below). Second, implementation of a rapid diagnostic assay, the FilmArray system V1.7 (BioFire/BioMerieux, UT, USA), performing the respiratory panel (RP) according to the manufacturer's protocol. The assay generates results within $70 \mathrm{~min}$ and includes 17 viral targets and three bacterial targets [11]. Third, extending the diagnostic service to 7 days a week from 8.30 am to 10 pm, between mid-December 2014 and early April 2015. This was continued in later respiratory seasons. 


\section{Patient selection \& testing}

Adult patients (18 years and older) presenting themselves at the ED of our tertiary care hospital (1339 beds and a total of $\sim 35,000 \mathrm{ED}$ visits each year), were included in this study when they were identified with acute respiratory illness, pneumonia, exacerbations of underlying conditions like asthma, COPD or similar, and when they had two or more of the following symptoms that were indicative for a respiratory viral infection: cough, cold, sore throat, temperature above $38^{\circ} \mathrm{C}$ or general signs of fever. During the respiratory season of 2014-2015, from mid-December to early April, a total of 641 diagnostic tests were requested by the ED, of which 492 complete sets of data were available for analysis. Median age of this group was 62 years old (IQR: 49-72) and gender distribution was 49\% females and $51 \%$ males.

Time of patient registration at the ED and time of diagnostic request were recorded - indicating the TAT at the ED. The time of the test results subsequently indicated the TAT at the clinical virology laboratory. This included order entry, performing the test and communication of the results to the treating physician at the ED.

Patients tested positive for a pathogen could be admitted to a ward with virus-specific cohorts. This represents a diagnostic and logistic scenario, in order to prevent potential spreading of a virus, without occupying single rooms. Especially in the respiratory season with an increased number of influenza virus A detections (type H3N2 or H1N1), this method aims to increase the bed capacity and to improve the patient flow within the hospital. The number of isolation events that could be avoided due to the rapid feedback of negative test results were analyzed, as well as the impact on decisions for hospitalization and prescribing antibiotic or antiviral therapy.

The very few patients admitted to the hospital between $10 \mathrm{pm}$ and 08.30 am were not specifically targeted for this new rapid policy, because this would be very expensive for relatively small benefits, but these patients remained included for calculating TAT, reflecting daily clinical practice.

Additionally, a selection of 260 samples that were initially tested negative with the FilmArray RP, were retested with our laboratory developed test (LDT) to calculate the negative predictive value (NPV).

\section{The euro-hour concept}

The €h concept was applied and €h was calculated by multiplying the costs with the total TAT [3]. This under the premise that rapidness in identification leads to earlier decision making and safer healthcare. Costs of safer healthcare is therefore a product of the laboratory costs (no additional costs are envisaged for the ED and were therefore be neglected in the equation) multiplied by the time to reporting of the result. This was done for the 'former' diagnostic policy using the LDT, as well as for the 'new' diagnostic policy using the rapid test (FilmArray $\mathrm{RP})$. The LDT was a multiplex real-time PCR assay and included the same viral targets as the FilmArray RP [11]. The LDT result also included a Ct value as a relative measure of viral load, and distinguishes between enterovirus and rhinovirus and between RSV A and RSV B. No bacterial targets were included in the LDT.

Based on internally available data, the costs for performing a multiplex LDT ranged from €200 to €240 per sample. This included labor costs, costs for reagents and run controls, depreciation and maintenance of equipment, external quality control as well as $20 \%$ overhead. In the new diagnostic policy with the use of the FilmArray RP, these costs increased, ranging from $€ 250$ to $€ 300$ per result, which also included additional labor costs for the extended working hours.

Based on a LEAN project at the ED, the mean total TAT of the former diagnostic policy was known to be $36 \mathrm{~h}$ : $17 \mathrm{~h}$ between the request at the ED and the start of diagnostic processing and subsequently $19 \mathrm{~h}$ at the laboratory (of which $28 \%$ of the results within $5 \mathrm{~h}$ and $72 \%$ within $24 \mathrm{~h}$ ). The relatively long TAT was due to the batch-wise approach and a nonoperational laboratory during the evening and weekends.

\section{Statistics}

The median with interquartile ranges (IQR) were used to express TAT of the new policy. To compare the new versus the former policy, mean TAT were available.

\section{Results}

An extensive and long respiratory season was observed, started at week 51, 2014, with an increasing number of influenza A virus-type H3N2-positive samples during the following 2 months. While the numbers of influenza A virus positives slowly dropped, we observed an increasing number of human rhinovirus (HRV), human metapneumovirus (hMPV), coronavirus (CoV)-OC43, CoV-229E, CoV-NL63 and RSV, starting from week 5 to week 13, 2015 (Figure 2). 


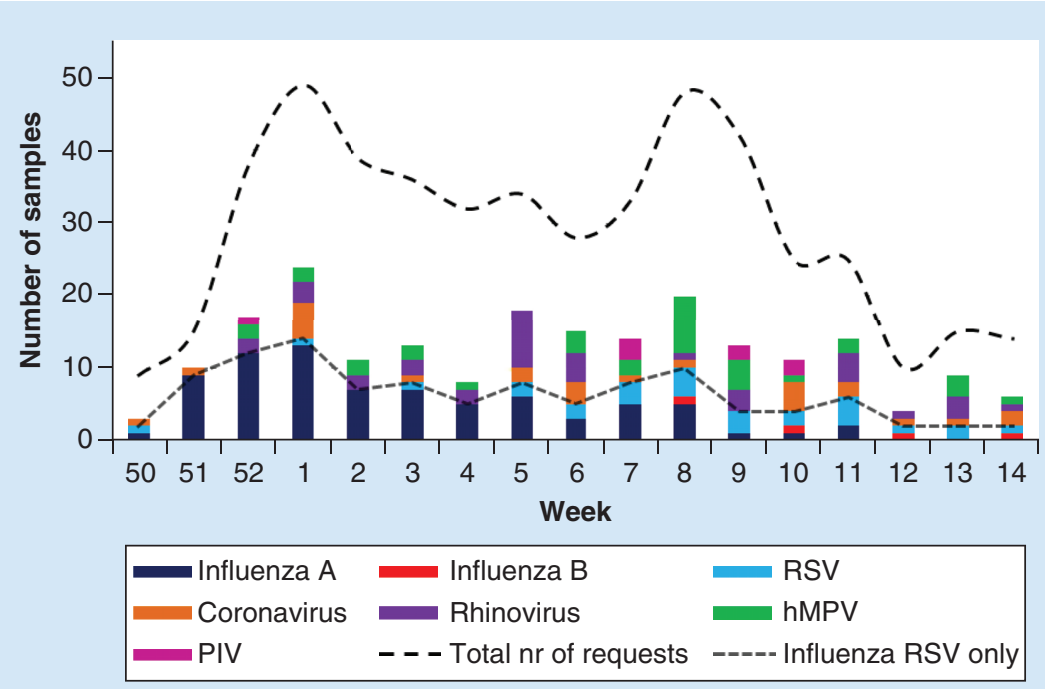

Figure 2. Number of requests (upper dashed line in bold) and infections (colored bars) that were detected with the FilmArray RP during the respiratory season in 2014-2015, aligned per week (Monday-Sunday). The lower dashed line shows the positivity rate when a syndromic approach will not be applied - influenza and RSV only.

\begin{tabular}{|c|c|c|}
\hline Virus & $\mathbf{N}$ & $\%$ \\
\hline Adenovirus & 0 & 0.0 \\
\hline Coronavirus 229E & 8 & 3.7 \\
\hline Coronavirus HKU1 & 0 & 0.0 \\
\hline Coronavirus OC43 & 10 & 4.7 \\
\hline Coronavirus NL63 & 7 & 3.3 \\
\hline Human metapneumovirus & 33 & 15.3 \\
\hline Human rhinovirus/enterovirus & 36 & 16.7 \\
\hline Influenza A & 6 & 2.8 \\
\hline Influenza A H1 & 0 & 0.0 \\
\hline Influenza A H1-2009 & 8 & 3.7 \\
\hline Influenza A H3N2 & 68 & 31.6 \\
\hline Influenza B & 5 & 2.3 \\
\hline Influenza total & 87 & 40.5 \\
\hline Parainfluenza 1 & 0 & 0.0 \\
\hline Parainfluenza 2 & 0 & 0.0 \\
\hline Parainfluenza 3 & 6 & 2.8 \\
\hline Parainfluenza 4 & 1 & 0.5 \\
\hline RSV & 27 & 12.6 \\
\hline Total viruses detected & 215 & 100 \\
\hline Samples tested positive & 207 & 42.1 \\
\hline Samples tested negative & 285 & 57.9 \\
\hline Total samples tested & 492 & 100 \\
\hline
\end{tabular}

The 492 diagnostic requests that were available for analysis resulted in the detection of 215 pathogens in 207 (42\%) samples and $285(58 \%)$ samples tested negative for any of the targets. Out of the 215 positive samples, 87 (41\%) were influenza, 27 (13\%) RSV and $101(47 \%)$ were different viral pathogens. Detection rates are shown in Table 1. Co-infections were observed in eight patients and consisted of four patients with both rhinovirus and influenza A virus-type H3N2, two patients had a coinfection of CoV-NL63 and CoV-OC43, one patient had a 
Table 2. Patient characteristics.

\begin{tabular}{|llll|}
\hline Parameters & Males & Females & Total \\
\hline Patients, $\mathrm{n}(\%)$ & $253(51)$ & $239(49)$ & 492 \\
\hline Mean age & 60 & 58 & 59 \\
\hline Median age & 62 & 61 & 62 \\
\hline IQR of age & $50-72$ & $48-71$ & $49-72$ \\
\hline Range of age & $18-94$ & $18-97$ & $18-97$ \\
\hline IQR: Interquartile range. & & & \\
\hline
\end{tabular}

coinfection of CoV-E229 and HRV and one patient had a coinfection of hMPV and HRV. Age and sex distribution is shown in Table 2 .

Six samples showed initially an influenza A virus 'equivocal' result, of which three were confirmed by the LDT and three were negative after repeating the test (with LDT and/or FilmArray RP). Additionally, five results were 'invalid', of which two samples were positive for RSV, one for hMPV, one for HRV and one for influenza A virus type H3N2. Though, these five results were confirmed using the LDT.

Furthermore, $30(12 \%)$ of the 260 samples that were initially tested negative using the FilmArray RP, were retested positive by the LDT assay for the following 31 viruses: $12 \mathrm{HRV}$, four influenza A virus, four CoV-229E, four CoV-OC43, three hMPV, two RSV B, one CoV-NL63 and one was enterovirus. Five of these had a $\mathrm{Ct}$ value of 27-30, eight results had a Ct value of 31-35 and the other 18 results had a Ct value of $>35$. This sets the negative predictive value (NPV) of the FilmArray RP at $88 \%$, important when negative test results are used for preventing isolation measures.

\section{Improved diagnostic policy \& TAT}

The median TAT at the ED (patient registration, sampling and dispatch sample to the laboratory) was 45 min (IQR: 31-82). 62\% of all samples were dispatched for diagnostic testing within $1 \mathrm{~h}$ and $86 \%$ within $2 \mathrm{~h}$ (Figure $3 \mathrm{~A}$ ). The median TAT of testing at the clinical laboratory was $110 \mathrm{~min}$ (IQR: 95-130). 59\% of all results were obtained within $2 \mathrm{~h}$ and $93 \%$ within $3 \mathrm{~h}$ after the request had been received (Figure 3B). The TAT did not show differences during any part of the day (data not shown). Four results were obtained after $12 \mathrm{~h}$; three of these were due to the late patient registration after $09.30 \mathrm{pm}$, when the laboratory service was already closed. This resulted in a total median TAT - from patient admission at the ED to the time of returning the result to the clinician at the ED - of $165 \mathrm{~min}$ (IQR: 138-214). 59\% of all results were obtained within $3 \mathrm{~h}$ and $83 \%$ within $4 \mathrm{~h}$ (Figure 3C).

The median length of stay of patients at the ED within our study population was $251 \mathrm{~min}$ (IQR 202-311 min). Because of this, 436 out of 492 (89\%) test results were communicated to the clinician while the patient was still at the ED.

\section{Diagnostic stewardship: hospitalization, patient isolation \& antimicrobial therapy}

When examining the decision for hospitalization, 153 out of the 492 (31\%) patients were not admitted, of which $66(43 \%)$ were tested positive for a viral target. 330 (67\%) patients were admitted to a clinical department in our hospital and nine (2\%) patients were re-directed to a regional hospital. Within this group of 339 admitted patients, $141(42 \%)$ were tested positive for a respiratory viral target, a similar percentage as in the group of patients who were not admitted.

From the 207 patients that were tested positive for a viral pathogen, 111 (54\%) received antibiotics, 94 (45\%) did not receive antibiotics and for two patients (1\%) data were not available. In the 86 patients that were tested positive for influenza, 48 (56\%) received oseltamivir, 37 (43\%) did not receive oseltamivir and for one patient $(1 \%)$ data were not available. These results indicate that the rapid diagnostic test result is not the critical parameter for the decision to admit a patient and to prescribe antimicrobial therapy.

Furthermore, 185 out of the 197 (94\%) negative test results were communicated to the clinician before the patient was admitted to a clinical department in our tertiary care hospital. Hence, admission with isolation measures because of a suspected viral infection, could be prevented in these 185/330 (56\%) admitted patients. 
(A)

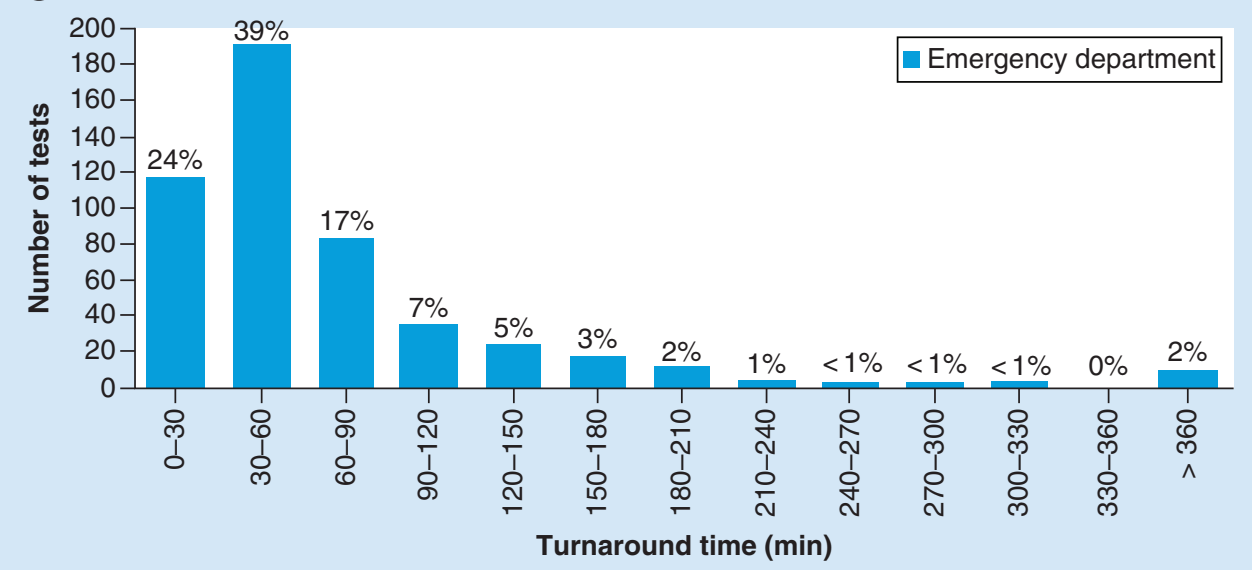

(B)

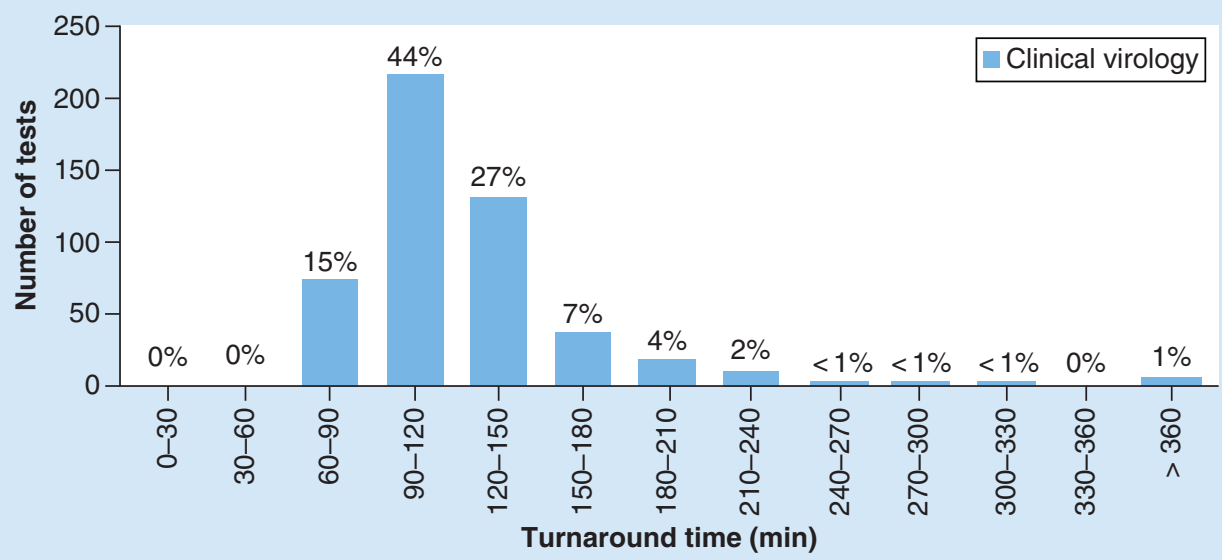

(C)

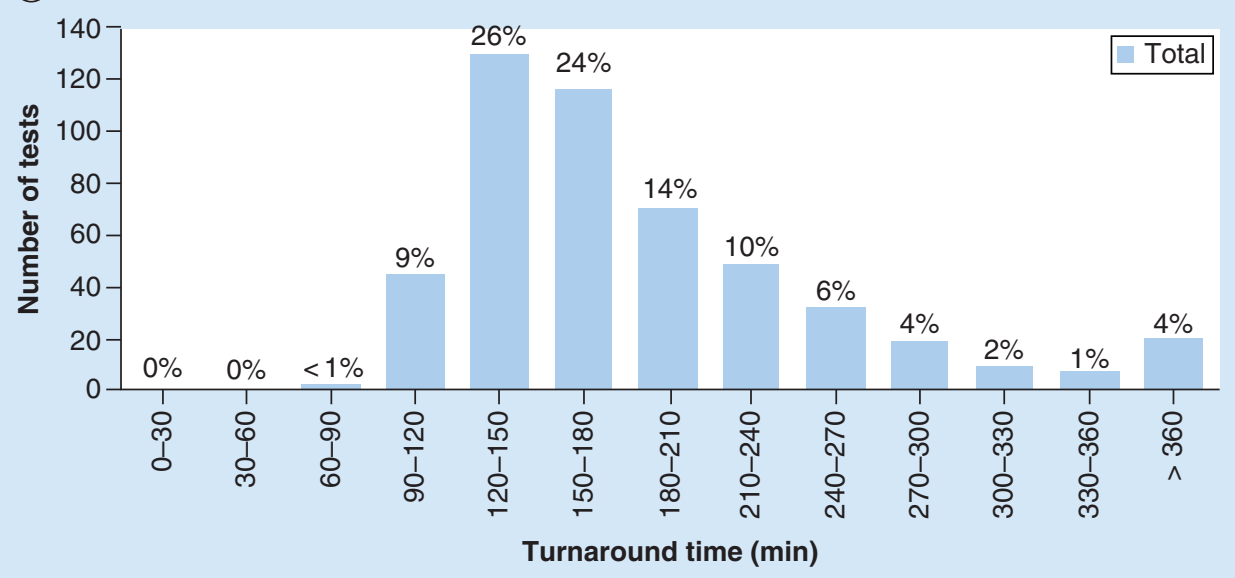

Figure 3. The number of diagnostic tests with a certain turnaround time (TAT), per 30 minutes. (A) Represents the TAT in the emergency department, (B) the TAT of testing in the clinical laboratory, and (C) the total TAT.

The euro-hour (€h) concept

The mean TAT at the ED (patient registration, sampling and dispatch sample to the laboratory) was reduced from $17 \mathrm{~h}$ to $1 \mathrm{~h}$ and $12 \mathrm{~min}(1.2 \mathrm{~h})$. The mean diagnostic TAT at the clinical virology laboratory was reduced from $19 \mathrm{~h}$ to $2 \mathrm{~h}$ and $2 \mathrm{~min}(2.03 \mathrm{~h})$. This resulted in a reduction of the total mean TAT from $36 \mathrm{~h}$ with the former 
Table 3. Euro-hour approach.

\begin{tabular}{lllll}
\hline Perspective & Policy & Costs per result $(€)$ & $\sim$ TAT (h) & $\begin{array}{l}\text { Impact of new vs former } \\
\text { policy }\end{array}$ \\
$\begin{array}{llll}\text { Diagnostic laboratory } \\
\text { (clinical virology) }\end{array}$ & 'Former' (PCR) & $200-240$ & 19 & $3800-4560$ \\
$\begin{array}{l}\text { Diagnostic laboratory and } \\
\text { emergency department }\end{array}$ & New (FA) & $250-300$ & 2.03 & $508-609$ \\
\hline
\end{tabular}

diagnostic policy, to $3 \mathrm{~h}$ and $14 \mathrm{~min}(3.23 \mathrm{~h})$ with the new diagnostic policy. By applying the €h concept and taking the perspective of the improved mean TAT at the diagnostic laboratory, the impact of the new diagnostic stewardship was increased with a factor of 6.2-9.0 (Table 3). When the perspective of the improved mean TAT at the ED is also included in the calculation, the impact of the new diagnostic stewardship was increased more, with a factor of 7.4-10.7 (Table 3).

\section{Discussion}

Challenges in triage processes and logistics do continuously arise at the ED. During the respiratory season, the ED's have a high patient burden [15]. Usually it takes several hours to find an available location for the patient, which is inconvenient and also very costly. This has a negative impact on patient care and the patient flow within and between hospitals and admission stops or closing of hospital wards are unfortunately frequent events. This stresses the importance of having a proper bed management system, supported by an adequate diagnostic test and diagnostic policy to optimize the patient flow during hospital admission. In this study, we described the impact of implementing a rapid diagnostic assay in the clinical virology laboratory, for the detection of respiratory viruses in patients at the ED, within a new, hospital-wide diagnostic policy.

A focus only on targets that historically have been considered as important like influenza or RSV, showed that the respiratory season would have ended around week 8 . However, since the number of requests from the ED did not decline significantly until week 12 - the sample number even increased again during week 7-9 - the new diagnostic service was extended to week 15 . This shows the importance of diagnostic testing with a syndromic approach, with assays that are not only targeted at influenza and/or RSV.

It is a general assumption that rapid diagnostic results by means of appropriate diagnostic stewardship adapted to the local and regional epidemiological situation, implies clinical benefits for patient care. Our data showed that the TAT was significantly reduced as a result of three main measures. First, sample collection in the ED was brought forward during triage, immediately after patient registration. While previously this decision was made after the patient was at the ED for a certain period, sometimes even just before admission to a hospital ward. The second measure was extending the diagnostic service of the clinical virology laboratory to $10 \mathrm{pm}$, including the weekend. Finally, introducing a rapid diagnostic assay. In our setting, the location of the clinical laboratory is in the vicinity of the ED. The impact was not only improved by decreasing the TAT of the assay itself, but rather by the newly introduced policy, encompassing the whole diagnostic routing.

Interestingly, the rapid test results were in many cases not the critical parameter for the decision of hospitalization or prescribing antimicrobial therapy within this patient population. One of the reasons to start antibiotic therapy in patients that were tested positive for a viral pathogen, was a possible bacterial superinfection. This is crucial information for assessing the value of the performed (rapid) diagnostics. In contrast, this study revealed that during the respiratory season 56\% (185/330) of the hospitalized patients did not need admission with isolation measures. They were tested negative before admission to a hospital ward. Generally, this implies a reduction of minimal one or two isolation days per patient, a significant benefit for the hospital wards as well as the continuously crowded ED. And because more beds are available for other patients, it has also an important financial benefit.

The negative predictive value (NPV) is also an important quality indicator regarding patient management and prevention of nosocomial infections. The quality of new commercial syndromic assays - often expressed in sensitivity and specificity - seems to approach the performance criteria of LDTs [16-19]. Although, the NPV that we calculated (88\%) indicates that innovative diagnostics still needs to be critically evaluated before implementation, irrelevant whether CE-IVD marked, US FDA approved or CLIA waived.

The FilmArray RP does not provide quantitative information. In our study however, samples are often obtained in patients with an acute respiratory infection at the ED, when samples most likely have a high viral load. 
TAT, performance indicators and costs are important parameters that defines the diagnostic value of an assay. From a managerial point of view, it is the responsibility of each healthcare institution to gain knowledge on the affordability of innovative diagnostics. Therefore, to assess the benefits of diagnostic testing, data such as reduced number of nosocomial infections, length of stay, isolation days or hospital admissions must be considered. Unfortunately these (clinical and financial) benefits are difficult to measure and in many cases even hardly available in The Netherlands, or data is not transparent, comparative or representative. Therefore, we conceptualized the euro-hour model [3]. This model might not be optimal, but it approaches the impact of a diagnostic test in patient care by multiplying costs of the assay by the TAT. In that perspective, the TAT reflects upon the clinical benefits including patient safety-related impact of a test result. The new diagnostic policy in this study showed a slight increase of costs, but also a huge decrease of the total TAT. Using the th concept, this has led up to a tenfold increase of the impact, compared with the previous diagnostic policy.

In 2016, we introduced a middleware solution called Flow point-of-care or FlowPOC, that improves the overall (digital) communication. The expected time-to-result is sent to the digital patient file and after the test is finished, the result becomes automatically visible, including links to several protocols (e.g., influenza treatment and isolation protocols).

\section{Conclusion}

In conclusion, diagnostic stewardship is crucial for creating an optimal hospital-broad diagnostic policy, in a cocreative manner [20]. Making use of the th concept increases awareness, improves the understanding of previous non-transparent outcomes (for any stakeholder) and attributes added value to patient safety by including rapidity of TAT in the cost calculation. An improved diagnostic service which is related to slight increased (direct) costs for the clinical virology laboratory should be waged against improved outcomes, mostly regarding the patient flow within the hospital, and financial benefits obtained in the ED and other clinical departments. This is important since incentives for innovation are limited in many routine healthcare settings due to rigid and complex financing structures that exist.

\section{Future perspective}

Rapid (commercial) diagnostic assays, so-called POC tests, are developed for implementation in various clinical settings and become readily available. No difficult procedures must be performed which seems very useful in certain settings like the $\mathrm{ED}$ of a hospital or when no laboratory with qualified staff is present.

The introduction of many of these assays will influence the system as a whole and might change the view on why and how diagnostics should be applied. This, however, differs per setting and should be reviewed by each medical professional that uses diagnostics for treatment of their patients or for patient management. The medical microbiology field must stay critical toward important parameters that define the value of a test, like TAT, the spectrum of targets in one test, quality indicators, finances and more. This also means that there should be a broader view than only the diagnostic parameters. A good laboratory-developed test with high quality which is embedded in a relatively poor (slow, not effective) diagnostic policy, will not affect treatment or patient management. By only implementing a rapid (commercial) test, this will not change. The policy should fit the diagnostic service as well. This is similar for any promising diagnostics that will be available soon.

\section{Author contributions}

R Poelman and HGM Niesters: substantial contribution to design/concept of the work, analysis and interpretation of data, drafting and revising the work, approved final version to be published and accountable for all aspects of the work. J van der Meer, $C$ van der Spek, A Riezebos-Brilman, M Knoester, C Van Leer-Buter and AW Friedrich: substantial contribution to design/concept of the work, interpretation of data, revising the work critically, approved final version to be published and accountable for all aspects of the work.

\section{Acknowledgments}

The authors thank LFR Span (Hematology), FLH Muntinghe (Internal Medicine), TJ Olgers (Internal Medicine), WR Douma (Pulmonary Diseases), EAM Verschuuren (Pulmonary Diseases) and HAM Kerstjens (Pulmonary Diseases), for their active participation and contribution in creating the optimal diagnostic policy. 
Financial \& competing interests disclosure

The authors have no relevant affiliations or financial involvement with any organization or entity with a financial interest in or financial conflict with the subject matter or materials discussed in the manuscript. This includes employment, consultancies, honoraria, stock ownership or options, expert testimony, grants or patents received or pending, or royalties.

No writing assistance was utilized in the production of this manuscript.

Ethical conduct of research

The authors state that they have obtained appropriate institutional review board approval or have followed the principles outlined in the Declaration of Helsinki for all human or animal experimental investigations.

\section{Open access}

This work is licensed under the Attribution-NonCommercial-NoDerivatives 4.0 Unported License. To view a copy of this license, visit http://creativecommons.org/licenses/by-nc-nd/4.0/

\section{Summary points}

- An optimal diagnostic policy for patients with respiratory viral infections supports the implementation of a rapid diagnostic test, the FilmArray respiratory panel. Laboratory turnaround time (TAT) is part of the total TAT, logistics are equally important as well.

- Diagnostic stewardship is a manner to use proper diagnostic testing for any therapy- and infection prevention-related clinical management of patients.

- Collaboration of medical microbiology staff with the emergency department and clinical departments were key to get to an optimal diagnostic policy.

- The mean turnaround time at the ED was decreased from $17 \mathrm{~h}$ to $1 \mathrm{~h}$ and $12 \mathrm{~min}$. The mean laboratory turnaround time from $19 \mathrm{~h}$ to $2 \mathrm{~h}$ and $2 \mathrm{~min}$. Thus, in total from $36 \mathrm{~h}$ to $3 \mathrm{~h}$ and $14 \mathrm{~min}$, a more than tenfold decrease.

- Syndromic testing revealed the complete picture of respiratory viral infections, detection rates showed that more than $50 \%$ of the positive samples were not influenza, still with a clinical picture that required the performance of a diagnostic test. These were RSV, human metapneumovirus, rhinovirus/enterovirus and coronavirus. Our study showed that this is important for preventing nosocomial infections.

- For patient management, a negative test result is as important as a positive test result. In this study, admission into an isolation room because of a suspected viral infection, could be prevented in 185/330 (56\%) patients admitted to our tertiary care hospital.

- Laboratories must stay critical towards the quality of (new, commercial) diagnostic assays. Especially regarding patient management, which relies on the negative test result of such a diagnostic assay, the negative predictive value (NPV) is reflecting the reliability of the assay when used for isolation measures. The NPV for the FilmArray $\mathrm{RP}$ in this study was $88 \%$.

- While direct clinical benefits due to the implementation of a rapid syndromic test are difficult to measure, the euro-hour (€h) concept helps to determine the value of a test with respect to patient safety. Using this concept, the impact of the new diagnostic policy was up to ten-times higher than the former policy.

- $45 \%$ of the patients that were tested positive for a viral pathogen, did not receive antibiotic therapy. $56 \%$ of the patients with influenza virus received antiviral therapy (oseltamivir). When diagnostic results are guiding antimicrobial therapy more routinely, these percentages could be improved.

- All these bullet points together explain why (tertiary care) hospitals need to have proper antimicrobial, infection prevention and diagnostic (AID) stewardship programs in place, for the benefit of the patient.

\section{References}

Papers of special note have been highlighted as: $\bullet$ of interest; $\bullet \bullet$ of considerable interest

1. Caliendo AM, Gilbert DN, Ginocchio CC et al. Better tests, better care: improved diagnostics for infectious diseases. Clin. Infect. Dis. 57(Suppl. 3), S139-S170 (2013).

2. Uyeki TM, Bernstein HH, Bradley JS et al. Clinical Practice Guidelines by the Infectious Diseases Society of America: 2018 update on diagnosis, treatment, chemoprophylaxis, and institutional outbreak management of seasonal influenza. Clin. Infect. Dis. 68(6), e1-e47 (2019).

3. Dik J-WH, Poelman R, Friedrich AW et al. An integrated stewardship model: antimicrobial, infection prevention and diagnostic (AID). Future Microbiol. 11(1), 93-102 (2016).

-• Gives a fundamental perspective of diagnostic stewardship, integrated with antimicrobial and infection prevention stewardship. A model that is aimed to serve as blueprint for implementing innovative integrative infection management. 
4. Poelman R, Schuffenecker I, Van Leer-Buter C et al. European surveillance for enterovirus D68 during the emerging North-American outbreak in 2014. J. Clin. Virol. 71, 1-9 (2015).

5. Knoester M, Helfferich J, Poelman R et al. Twenty-nine cases of Enterovirus-D68 associated acute flaccid myelitis in Europe 2016; a case series and epidemiologic overview. Pediatr. Infect. Dis. J. 38(1), 16-21 (2018).

6. McAllister SC, Schleiss MR, Arbefeville S et al. Epidemic 2014 enterovirus D68 cross-reacts with human rhinovirus on a respiratory molecular diagnostic platform. PLoS ONE 10(3), e0118529 (2015).

- Reveals the importance of staying critical towards the quality of new diagnostic platforms. Rhinoviruses could be detected on the platform while representing enterovirus D68.

7. Echavarría M, Marcone DN, Querci M et al. Clinical impact of rapid molecular detection of respiratory pathogens in patients with acute respiratory infection. J. Clin. Virol. 108, 90-95 (2018).

8. Vos LM, Riezebos-Brilman A, Schuurman R, Hoepelman AIM, Oosterheert JJ. Syndromic sample-to-result PCR testing for respiratory infections in adult patients. Neth. J. Med. 76(6), 286-293 (2018).

9. Rogers BB, Shankar P, Jerris RC et al. Impact of a rapid respiratory panel test on patient outcomes. Arch. Pathol. Lab. Med. 139(5), 636-641 (2015).

-• One of the first papers that describes the potential impact of a rapid diagnostic assay on patient outcomes for children admitted to the hospital.

10. Herr CEW, Heckrodt TH, Hofmann FA, Schnettler R, Eikmann TF. Additional costs for preventing the spread of methicillin-resistant Staphylococcus aureus and a strategy for reducing these costs on a surgical ward. Infect. Control Hosp. Epidemiol. 24(9), 673-678 (2003).

11. Butt SA, Maceira VP, McCallen ME, Stellrecht KA. Comparison of three commercial RT-PCR systems for the detection of respiratory viruses. J. Clin. Virol. 61(3), 406-410 (2014).

12. Rappo U, Schuetz AN, Jenkins SG et al. Impact of early detection of respiratory viruses by multiplex PCR assay on clinical outcomes in adult patients (Caliendo AM, ed.). J. Clin. Microbiol. 54(8), 2096-2103 (2016).

13. Brendish NJ, Schiff HF, Clark TW. Point-of-care testing for respiratory viruses in adults: the current landscape and future potential. $J$. Infect. 71(5), 501-510 (2015).

- Describes the diagnostic landscape and assesses the potential clinical benefits of point-of-care testing.

14. Nelson RE, Stockmann C, Hersh AL et al. Economic analysis of rapid and sensitive polymerase chain reaction testing in the emergency department for influenza infections in children. Pediatr. Infect. Dis. J. 34(6), 577-582 (2015).

-. Describes an economic analysis of rapid testing for influenza in the emergency department.

15. Muscatello D, Bein K, Dinh M. Emergency Department demand associated with seasonal influenza, 2010 through 2014, New South Wales, Australia. West Pacific Surveill. Response J. 8(3), 11-20 (2017).

16. Pierce VM, Elkan M, Leet M, McGowan KL, Hodinka RL. Comparison of the Idaho Technology FilmArray system to real-time PCR for detection of respiratory pathogens in children. J. Clin. Microbiol. 50(2), 364-371 (2012).

17. Salez N, Ninove L, Thirion L et al. Evaluation of the Xpert Flu test and comparison with in-house real-time RT-PCR assays for detection of influenza virus from 2008 to 2011 in Marseille, France. Clin. Microbiol. Infect. 18(4), E81-E83 (2012).

18. Andersson ME, Olofsson S, Lindh M. Comparison of the FilmArray assay and in-house real-time PCR for detection of respiratory infection. Scand. J. Infect. Dis. 46(12), 897-901 (2014).

19. Chen JHK, Lam H-Y, Yip CCY et al. Clinical evaluation of the new high-throughput Luminex NxTAG Respiratory Pathogen Panel assay for multiplex respiratory pathogen detection. J. Clin. Microbiol. 54(7), 1820-1825 (2016).

20. Messacar K, Parker SK, Todd JK, Dominguez SR. Implementation of rapid molecular infectious disease diagnostics: the role of diagnostic and antimicrobial stewardship. J. Clin. Microbiol. 55(3), 715-723 (2017).

•• Focuses on the role of diagnostic stewardship while implementing rapid molecular diagnostics for infectious disease. 